\title{
Response of the CALICE Si-W Electromagnetic Calorimeter Physics Prototype to Electrons
}

C. Adloff ${ }^{\mathrm{a}}$, Y. Karyotakis ${ }^{\mathrm{a}}$, J. Repond $^{\mathrm{b}}$, J. Yu $^{\mathrm{c}}$, G. Eigen ${ }^{\mathrm{d}}$, C. M.Hawkes ${ }^{\mathrm{e}}$, Y. Mikami $^{\mathrm{e}}$, O. Miller ${ }^{\mathrm{e}}$, N. K.Watson ${ }^{\mathrm{e}}$, J.A. Wilson ${ }^{\mathrm{e}}$, T. Goto ${ }^{\mathrm{f}}$, G. Mavromanolakis ${ }^{\mathrm{f}}$,

M.A. Thomson ${ }^{\mathrm{f}}$, D.R. Ward ${ }^{\mathrm{f}}$, W. Yan ${ }^{\mathrm{f}}$, D. Benchekroun ${ }^{\mathrm{g}}$, A. Hoummada ${ }^{\mathrm{g}}$, M. Krim${ }^{\mathrm{g}}$,

M. Benyamna ${ }^{\text {h }}$, D. Boumediene ${ }^{\text {h }}$, N. Brun ${ }^{\text {h }}$, C. Cârloganu, h, P. Gay ${ }^{\text {h }}$, F. Morisseau ${ }^{\text {h }}$, G. C.Blazey ${ }^{i}$, D. Chakraborty ${ }^{i}$, A. Dyshkant ${ }^{i}$, K. Francis ${ }^{i}$, D. Hedin ${ }^{i}$, G. Lima ${ }^{i}$, V. Zutshi ${ }^{i}$, J.-Y. Hostachy ${ }^{\mathrm{j}}$, L. Morin ${ }^{\mathrm{j}}$, N. D’Ascenzo ${ }^{\mathrm{k}}$, U. Cornett ${ }^{\mathrm{k}}$, D. David ${ }^{\mathrm{k}}$, R. Fabbrik ${ }^{k}$ G. Falley ${ }^{\mathrm{k}}$, K. Gadow ${ }^{\mathrm{k}}$, E. Garutti ${ }^{\mathrm{k}}$, P. Göttlicherk ${ }^{\mathrm{k}}$, T. Jungk

S. Karstensen ${ }^{\mathrm{k}}$, V. Korbel ${ }^{\mathrm{k}}$, A.-I. Lucaci-Timoce ${ }^{\mathrm{k}}$, B. Lutz ${ }^{\mathrm{k}}$, N. Meyer ${ }^{\mathrm{k}}$, V. Morgunov ${ }^{\mathrm{k}}$, M. Reinecke ${ }^{\mathrm{k}}$, F. Sefkow ${ }^{\mathrm{k}}$, P. Smirnov ${ }^{\mathrm{k}}$, A. Vargas-Trevino ${ }^{\mathrm{k}}$, N. Wattimena ${ }^{\mathrm{k}}$,

O. Wendt ${ }^{\mathrm{k}}$, N. Feege ${ }^{\mathrm{l}}$, M. Groll ${ }^{1}$, J. Haller ${ }^{1}$, R.-D. Heuer ${ }^{1}$, S. Richter ${ }^{1}$, J. Samson ${ }^{1}$,

A. Kaplan ${ }^{\mathrm{m}}$, H.-Ch. Schultz-Coulon ${ }^{\mathrm{m}}$, W. Shen ${ }^{\mathrm{m}}$, A. Tadday ${ }^{\mathrm{m}}$, B. Bilki ${ }^{\mathrm{n}}$, E. Norbeck $^{\mathrm{n}}$,

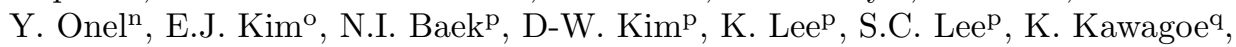

Y. Tamura ${ }^{\mathrm{q}}$, D.A. Bowerman ${ }^{\mathrm{r}}$, P.D. Dauncey ${ }^{\mathrm{r}}$, A.-M. Magnan ${ }^{\mathrm{r}}$, H. Yilmaz ${ }^{\mathrm{r}}$, O. Zorba ${ }^{\mathrm{r}}$, V. Bartsch ${ }^{\mathrm{s}}$, M. Postranecky ${ }^{\mathrm{s}}$, M. Warren ${ }^{\mathrm{s}}$, M. Wing ${ }^{\mathrm{s}}$, M. Faucci Giannelli ${ }^{\mathrm{t}}$,

M.G. Green ${ }^{\mathrm{t}}$, F. Salvatore ${ }^{\mathrm{t}}$, M. Bedjidian ${ }^{\mathrm{u}}$, R. Kieffer ${ }^{\mathrm{u}}$, I. Laktineh ${ }^{\mathrm{u}}$, D.S. Bailey ${ }^{\mathrm{v}}$,

R.J. Barlowv ${ }^{\mathrm{v}}$, M. Kelly ${ }^{\mathrm{v}}$, R.J. Thompson ${ }^{\mathrm{v}}$, M. Danilovw, E. Tarkovskyw , N. Baranova ${ }^{\mathrm{x}}$,

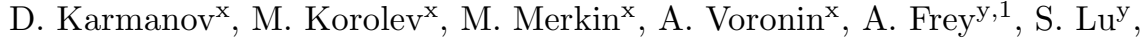

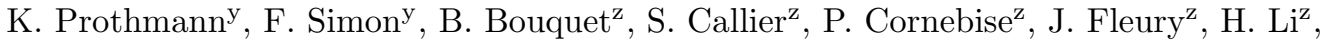
F. Richard ${ }^{\mathrm{z}}$, Ch. de la Taille, R. Poeschl ${ }^{\mathrm{z}}$, L. Raux ${ }^{\mathrm{z}}$, M. Ruan ${ }^{\mathrm{z}}$, N. Seguin-Moreau ${ }^{\mathrm{z}}$, F. Wicek ${ }^{z}$, M. Anduze ${ }^{\text {aa }}$, V. Boudry ${ }^{\text {aa }}$, J-C. Brient ${ }^{\text {aa }}$, G. Gaycken ${ }^{\text {aa }}$, P. Mora de Freitas $^{\text {aa }}$, G. Musat ${ }^{\text {aa }}$, M. Reinhard ${ }^{\text {aa }}$, A. Rougéa ${ }^{a}$, J-Ch. Vanel $^{\text {aa }}$, H. Videau ${ }^{\text {aa }}$, K-H. Park ${ }^{\text {ab }}$, J. Zacek ${ }^{\text {ac }}$, J. Cvach ${ }^{\text {ad }}$, P. Gallus ${ }^{\text {ad }}$, M. Havranek ${ }^{\text {ad }}$, M. Janata ${ }^{\text {ad }}$, M. Marcisovsky ${ }^{\text {ad }}$, I. Polak ${ }^{\text {ad }}$, J. Popule $^{\text {ad }}$, L. Tomasek $^{\text {ad }}$, M. Tomasek ${ }^{\text {ad }}$, P. Ruzicka ${ }^{\text {ad }}$,

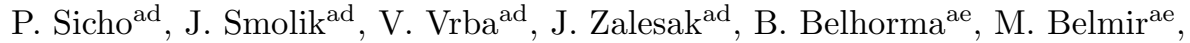

S. W.Nam ${ }^{\text {af }}$, I.H. Park ${ }^{\text {af }}$, J. Yang ${ }^{\text {af }}$, J.-S. Chai ${ }^{\text {ag }}$, J.-T. Kim ${ }^{\text {ag }}$, G.-B. Kim ${ }^{\text {ag }}$, J. Kang ${ }^{\text {ah }}$, Y.-J. Kwon ${ }^{\text {ah }}$

\footnotetext{
${ }^{a}$ Laboratoire d'Annecy-le-vieux de Physique des Particules, Chemin du Bellevue BP 110, F-74941 Annecy-le-Vieux Cedex, France

${ }^{b}$ Argonne National Laboratory, 9700 S. Cass Avenue, Argonne, IL 60439-4815, USA

${ }^{c}$ University of Texas, Arlington, TX 76019, USA

${ }^{d}$ University of Bergen, Inst. of Physics, Allegaten 55, N-5007 Bergen, Norway

${ }^{e}$ University of Birmingham, School of Physics and Astronomy, Edgbaston, Birmingham B15 2TT, UK

${ }^{f}$ University of Cambridge, Cavendish Laboratory, J J Thomson Avenue, CB3 OHE, UK

${ }^{g}$ Université Hassan II Ä̈n Chock, Faculté des sciences. B.P. 5366 Maarif, Casablanca, Morocco

${ }^{h}$ Laboratoire de Physique Corpusculaire de Clermont-Ferrand (LPC), 24 avenue des Landais, 63177 Aubière CEDEX, France

${ }^{i}$ NICADD, Northern Illinois University, Department of Physics, DeKalb, IL 60115, USA

${ }^{j}$ Laboratoire de Physique Subatomique et de Cosmologie - Université Joseph Fourier Grenoble 1 -

CNRS/IN2P3 - Institut Polytechnique de Grenoble, 53, rue des Martyrs, 38026 Grenoble CEDEX, France

${ }^{k}$ DESY, Notkestrasse 85, D-22603 Hamburg, Germany

${ }^{l}$ Univ. Hamburg, Physics Department, Institut für Experimentalphysik, Luruper Chaussee 149, 22761 Hamburg, Germany

${ }^{m}$ University of Heidelberg, Fakultat fur Physik und Astronomie, Albert Uberle Str. 3-5 2.OG Ost, D-69120 Heidelberg, Germany
} 
${ }^{n}$ University of Iowa, Dept. of Physics and Astronomy, 203 Van Allen Hall, Iowa City, IA 52242-1479, USA

${ }^{\circ}$ Chonbuk National University, Jeonju, 561-756, South Korea

${ }^{p}$ Kangnung National University, HEP/PD, Kangnung, South Korea

${ }^{q}$ Department of Physics, Kobe University, Kobe, 657-8501, Japan

${ }^{r}$ Imperial College, Blackett Laboratory, Department of Physics, Prince Consort Road, London SW7 $2 B W, U K$

${ }^{s}$ Department of Physics and Astronomy, University College London, Gower Street, London WC1E $6 B T, U K$

${ }^{t}$ Royal Holloway University of London, Dept. of Physics, Egham, Surrey TW20 OEX, UK

${ }^{u}$ Université de Lyon, F-69622, Lyon, France ; Université de Lyon 1, Villeurbanne ; CNRS/IN2P3, Institut de Physique Nucléaire de Lyon

${ }^{v}$ The University of Manchester, School of Physics and Astronomy, Schuster Lab, Manchester M13 9PL, UK

${ }^{w}$ Institute of Theoretical and Experimental Physics, B. Cheremushkinskaya ul. 25, RU-117218 Moscow, Russia

${ }^{x}$ M.V. Lomonosov Moscow State University, D.V.Skobeltsyn Institute of Nuclear Physics (SINP MSU), 1/2 Leninskiye Gory, Moscow, 119991, Russia

${ }^{y}$ Max Planck Inst. für Physik, Föhringer Ring 6, D-80805 Munich, Germany

${ }^{z}$ Laboratoire de L'accélerateur Linéaire, Centre d'Orsay, Université de Paris-Sud XI, BP 34 , Bâtiment 200, F-91898 Orsay CEDEX, France

${ }^{a a}$ École Polytechnique, Laboratoire Leprince-Ringuet (LLR), Route de Saclay, F-91128 Palaiseau, CEDEX France

${ }^{a b}$ Pohang Accelerator Laboratory, Pohang 790-784, South Korea

${ }^{a c}$ Charles University, Institute of Particle 83 Nuclear Physics, V Holesovickach 2, CZ-18000 Prague 8, Czech Republic

${ }^{a d}$ Institute of Physics, Academy of Sciences of the Czech Republic, Na Slovance 2, CZ-18221 Prague 8, Czech Republic

${ }^{a e}$ Centre National de l'Energie, des Sciences et des Techniques Nucléaires, B.P. 1382, R.P. 10001, Rabat, Morocco

${ }^{a f}$ Ewha Womans University, Dept. of Physics, Seoul 120, South Korea

${ }^{a g}$ Sungkyunkwan University, 300 Cheoncheon-dong, Jangan-gu, Suwon, Gyeonggi-do 440-746, South Korea

${ }^{a h}$ Yonsei University, Dept. of Physics, 134 Sinchon-dong, Sudaemoon-gu, Seoul 120-749, South Korea

\begin{abstract}
A prototype Silicon-Tungsten electromagnetic calorimeter (ECAL) for an International Linear Collider (ILC) detector was installed and tested during summer and autumn 2006 at CERN. The detector had 6480 silicon pads of dimension $1 \times 1 \mathrm{~cm}^{2}$. Data were collected with electron beams in the energy range 6 to $45 \mathrm{GeV}$. The analysis described in this paper focuses on electromagnetic shower reconstruction and characterises the ECAL response to electrons in terms of energy resolution and linearity. The detector is linear to within approximately the $1 \%$ level and has a relative energy resolution of $(16.6 \pm 0.1) / \sqrt{E(\mathrm{GeV})} \oplus 1.1 \pm 0.1(\%)$. The spatial uniformity and the time stability of the ECAL are also addressed.
\end{abstract}

Key words:

CALICE, ILC, electromagnetic calorimeter, silicon detector, electron reconstruction 


\section{Introduction}

The CALICE Collaboration is conducting R\&D into calorimetric systems for the ILC [1] - a proposed $\mathrm{e}^{+} \mathrm{e}^{-}$linear collider intended to operate at a centre of mass energy ranging up to the $\mathrm{TeV}$ scale. The physics scope at the ILC includes precise measurements of the triple- and quartic-gauge bosons interactions, as well as the complete characterisation of the Higgs and top quark sectors. In addition, hints of physics beyond the Standard Model could be addressed in a model-independent way.

The final states are typically multiple hadronic jets, accompanied frequently by low momentum leptons and/or missing energy. In such cases, lepton identification is difficult and the signature of the final states of interest relies on the identification of $\mathrm{Z}$ or/and $\mathrm{W}$ bosons in their decay modes into two jets. In order to distinguish them efficiently, a jet energy resolution close to $30 \% / \sqrt{E / \mathrm{GeV}}$ has to be achieved [1]. A precise reconstruction of the jet direction is also required. These are the main requirements driving the detector design in general at the ILC and the calorimetry design in particular.

The target jet energy resolution represents an improvement by a factor of two over the best obtained in previous detectors. Moreover the detection environment becomes more complex with increasing centre of mass energy. A promising way to achieve this increase in resolution is through designing a detector system optimised for the so called "particle flow" approach [2], which relies on the separate reconstruction of as many particles in the jet as possible, using the most suitable detector systems.

The success of such an algorithm depends on the quality of the pattern recognition in the calorimeters. For particle flow, a high spatial granularity is therefore as important as the intrinsic energy resolution for single particles. Furthermore, the overall design of the detector (tracking, electromagnetic and hadronic calorimetry) needs to be considered in a coherent way.

The design of the ILC detectors can be optimised using Monte Carlo simulations, but in order to do this, it is crucial to validate the Monte Carlo tools with data. Therefore, the R\&D of the CALICE Collaboration has two broad aims. The first is to construct realistic calorimeter prototypes, and learn about their operation and behaviour in beam tests. The second objective is to compare the data with Monte Carlo simulations using the same tools used for the full detector. This is especially important in the case of hadronic showers, where many models are available, which make differing predictions for the calorimeter response. The CALICE plan is to expose complete calorimeter systems (electromagnetic and hadronic, using various technologies) to test beams of electrons, muons and hadrons. To this end, a first round of beam tests was performed at DESY and CERN in summer 2006, using a Silicon-Tungsten sampling electromagnetic calorimeter [3], followed by a hadron calorimeter composed of iron and scintillator tiles [4], and then a Tail Catcher and Muon Counter (TCMT) of iron instrumented with scintillator strips [5].

In this paper, we report results of exposure of the prototype to electron beams in the energy range 6-45 GeV at the CERN H6 beam line [6]. In Section 2 we outline the layout of the beam tests. The ECAL is briefly described in Section 3 and some key technical aspects of its performance are highlighted. Section 4 summarises the Monte

\footnotetext{
*Corresponding author, e-mail: carlogan@in2p3.fr

${ }^{1}$ Now at Universität Göttingen
} 
Carlo simulation. Features of the electron beam data are reviewed in Section 5 and the uniformity across the detector is addressed. The results of the energy measurement together with some of their systematic uncertainties are presented in Section 6 for the detector areas of uniform response.

Several other studies of the prototype are ongoing, exploiting its unprecedentedly fine segmentation and capacity to observe shower development in detail. These studies will be reported in subsequent publications.

\section{Experimental Setup}

A sketch of the CERN H6 [6] test beam setup is presented in Figure 1 and a detailed description of the detectors can be found in [3]. The coordinate system used is right handed. The surface of the drift chamber (DC1) closest to the ECAL defines the origin, the $z$ axis is the beam axis and $x$ and $y$ the horizontal and upward-vertical, respectively.

The physics program and the overall electron, pion and muon statistics collected are extensively discussed in [3]. The beam trigger was defined by the coincidence signal of two scintillator counters. In addition, three drift chambers were used to monitor the beam. A threshold Čerenkov detector was also available for $e / \pi$ discrimination.

This paper presents the measurement of the ECAL response to electrons normally incident on the calorimeter surface. The event display for one of these events is shown in Figure 2, where the energy of the hits is measured in MIP units, one MIP being the signal left by a minimum ionising particle.

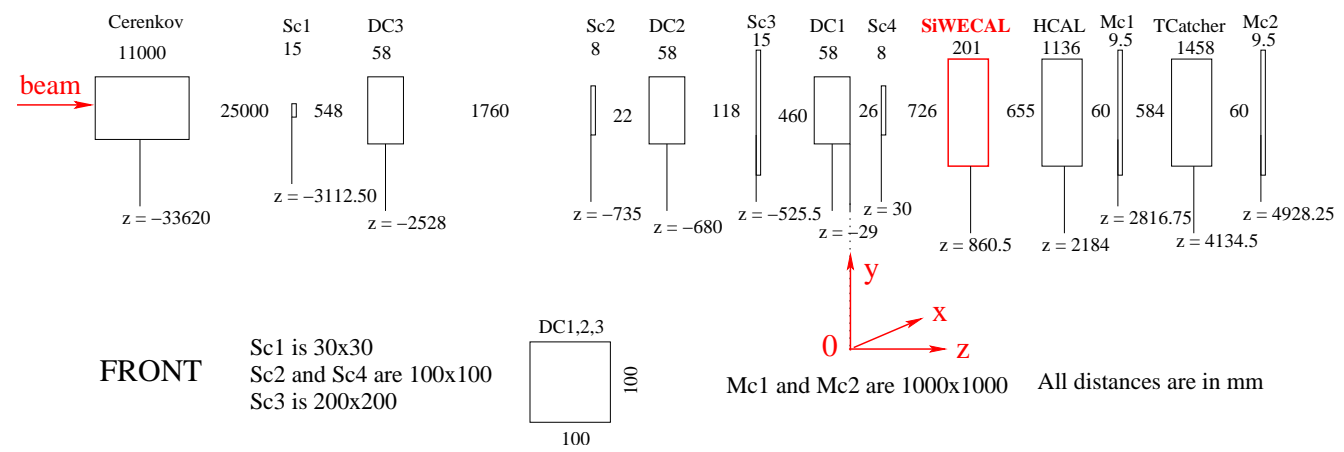

Figure 1: Sketch of the CERN test beam setup. The the right handed coordinate system used hereafter is indicated.

\section{The Si-W ECAL prototype}

A detailed description of the ECAL hardware is given in [3], along with details of the commissioning and a number of technical features of the system calibration and performance. The ECAL prototype consisted of 30 layers of tungsten, the first ten of thickness $1.4 \mathrm{~mm}$, the next ten of $2.8 \mathrm{~mm}$ and the last ten of $4.2 \mathrm{~mm}$, comprising 24 radiation lengths in total at normal incidence. The development of the showers was sampled using 30 layers of silicon PIN diode pads interleaved between the tungsten plates. 


\section{Run 300672:0 Event 1390}

Time: 04:53:16:523:075 Fri Oct 202006

Hits: 176 Energy: 1487.91 mips
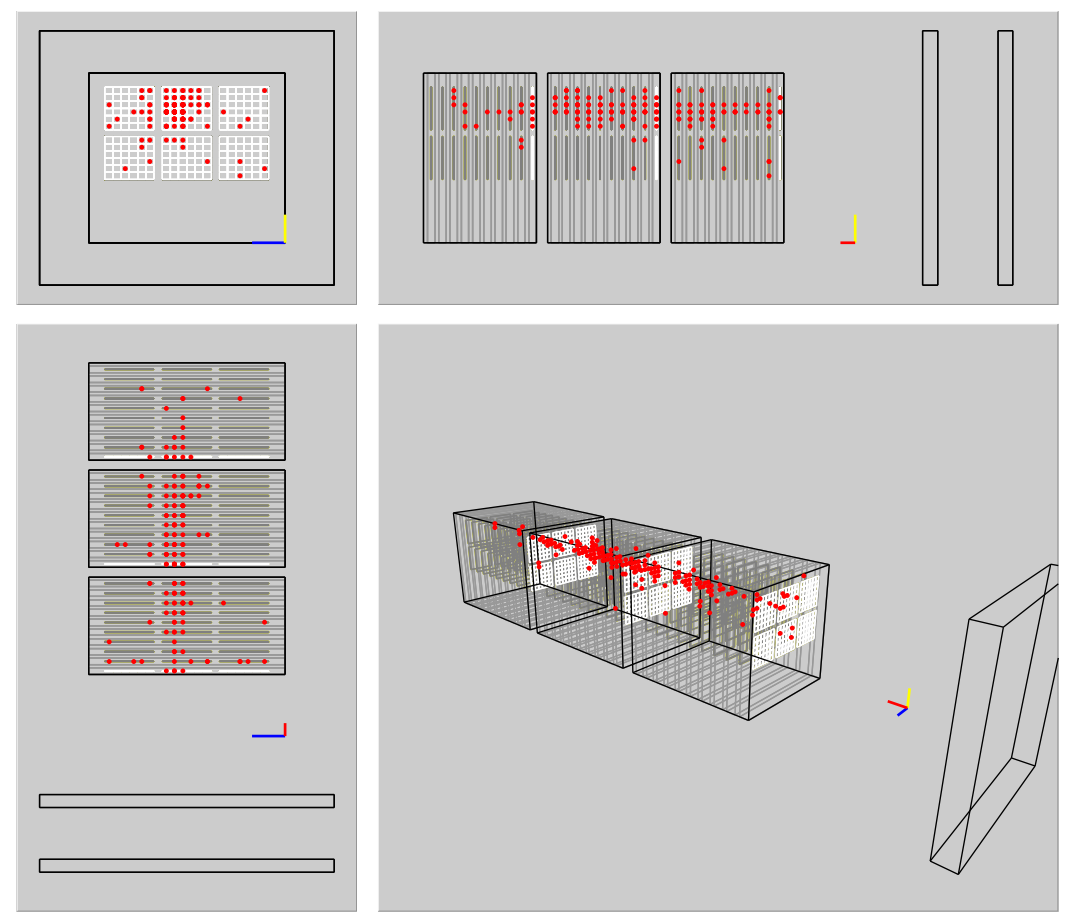

Figure 2: A typical $10 \mathrm{GeV}$ electron shower in the ECAL. The displayed detector cells have energies higher than 0.5 MIPs. Layout not to scale. 
The silicon thickness was $525 \mu \mathrm{m}$, with each pad having a transverse area of $1 \times 1 \mathrm{~cm}^{2}$. The sensors were implemented on 4 square inch wafers, segmented into $6 \times 6$ pads. At the time of the 2006 CERN beam tests, each layer consisted of a $3 \times 2$ array of wafers, i.e. 18 pads horizontally and 12 pads vertically, leading to a total of 6480 pads for the ECAL.

Blocks of random triggers were recorded during data taking in order to monitor pedestals and noise. Short term changes and shifts in pedestals caused by large signals in neighbouring cells were monitored and corrected using cells without signal in beam events [3]. The uncertainty on the pedestal levels was estimated to be less than 0.002 MIPs, negligible compared to the energy deposited by electron showers (a $10 \mathrm{GeV}$ electron deposits on average $1450 \mathrm{MIPs}$ ). The noise level was typically $0.13 \mathrm{MIPs}$; its spread channel-to-channel was $9 \%$ of the mean noise and the spread run-to-run was less than $1 \%$ of the mean noise. The low spread of the noise justifies the use of a single energy threshold for all cells in the detector.

Calibration constants for each pad were determined using muon events. The response of each cell was fitted by a convolution of a Landau distribution with a Gaussian. The most probable value of the underlying Landau function was taken to define the MIP value for each cell, and the raw energy for each cell in data was corrected to units of MIPs. All but 9 pads were functioning and successfully calibrated. The calibration constants were determined with an accuracy of $0.5 \%$ and had a cell to cell dispersion of $5 \%$. Data taken in the various beam test periods during summer and autumn 2006 showed well correlated calibration constants, with differences less than $1.6 \%$.

One feature of the data which has not been accounted for in the detector simulation is associated with showers which deposit a sizeable energy in the guard ring surrounding a wafer. This is a cause of correlated crosstalk, observed as a distinctive square pattern of low energy hits in a number of cells around the periphery of the wafer. The prevalence of this effect increases with the shower energy crossing the guard ring and therefore its rate is significantly reduced when considering only electrons impinging on the centre of the wafers. In the future, the design of the guard rings will be modified in order to prevent this problem.

After calibration, the ECAL data consist of hits in the cells of the calorimeter with energies in units of MIPs. In order to remove most of the noise signals, a threshold cut of 0.6 MIP was imposed on each cell, almost five times the mean noise level.

\section{Monte Carlo Simulation}

The test beam setup is simulated with Mokka 7], a Geant4 [8]-based Monte Carlo program, followed by a digitisation module simulating the response of the data acquisition electronics. The material upstream of the ECAL is taken into account. The subdetectors are simulated with different levels of detail, depending on their impact on the physics analysis: material simulation only for the Cerenkov detectors, raw energy depositions stored for the trigger counters, partial electronics simulation for the tracking detectors. In the case of the ECAL, the simulation gives the raw energy depositions in the Si pads and the readout electronics is simulated assuming that each channel exhibits only Gaussian noise. The mean values of the noise for each channel follow a Gaussian distribution over the ECAL, with the mean value of 0.13 MIPs and 0.012 MIPs dispersion, as measured. Since $99.9 \%$ of the ECAL cells were functioning, the impact of the non-responding cells 
is expected to be small and their signals were not supressed in the simulation for this analysis.

The beam simulation assumes a parallel beam with Gaussian width reproducing the observed beam profile. To study systematic effects due to lateral leakage of the showers, samples are also generated with a beam spread uniformily over the ECAL front face. A Gaussian momentum dispersion consistent with the settings of the beam collimators [6] is applied for each run.

\section{Selection of Electron Events}

Single electron showers are selected using the energy recorded in the ECAL. This energy, $E_{\text {raw }}$, is calculated with the three ECAL modules weighted in proportion to the tungsten thickness:

$$
E_{\text {raw }}=\sum_{i=0}^{i=9} E_{i}+2 \sum_{i=10}^{i=19} E_{i}+3 \sum_{i=20}^{i=29} E_{i},
$$

where $E_{i}$ is the energy deposit in layer $i$. The distribution of $E_{\text {raw }}$ is shown in Figure 3 for a typical $15 \mathrm{GeV}$ event sample. The electron peak at around 3900 MIPs is clearly visible; however, the muon and pion contamination in the beam gives an additional peak at 85 MIPs and the region between the two main peaks is populated with pions. Electron candidates are selected by requiring:

$$
125<\frac{E_{\text {raw }}(\mathrm{MIP})}{E_{\text {beam }}(\mathrm{GeV})}<375 .
$$

The significant pion contamination present in some of the data runs is reduced by demanding a trigger signal from the threshold Cerenkov counter in the beam. The effect of this additional requirement is indicated by the shaded region in Figure 3 .

\subsection{Rejection of the beam halo}

The rejection of the beam halo is implemented run-by-run. The $x$ and $y$ acceptance for the incoming electron track is chosen such as to achieve a reasonably flat distribution of the mean energy deposition in the ECAL.

\subsection{Inter-wafer gap effect}

Around the pads in each wafer, a non-active region of $1 \mathrm{~mm}$ width was used for a grounded guard ring structure. This creates a non-active gap between adjacent $\mathrm{Si}$ pads situated on different wafers $(2 \mathrm{~mm})$ which is significant compared to the transverse shower size. These non-active regions, called in the following "inter-wafer gaps" degrade the prototype response when showers traverse them. This is illustrated in Figure 4 for $30 \mathrm{GeV}$ electrons impinging on the calorimeter at normal incidence. Here the mean value of $E_{\text {raw }}$ (Equation 10 is plotted as a function of the shower barycentre $(\bar{x}, \bar{y})$, defined as :

$$
(\bar{x}, \bar{y})=\sum_{i}\left(E_{i} x_{i}, E_{i} y_{i}\right) / \sum_{i} E_{i}
$$

The sums run over all hit cells in the calorimeter. Dips in response corresponding to the guard ring positions are clearly visible: the energy loss is about $15 \%$ when electrons 


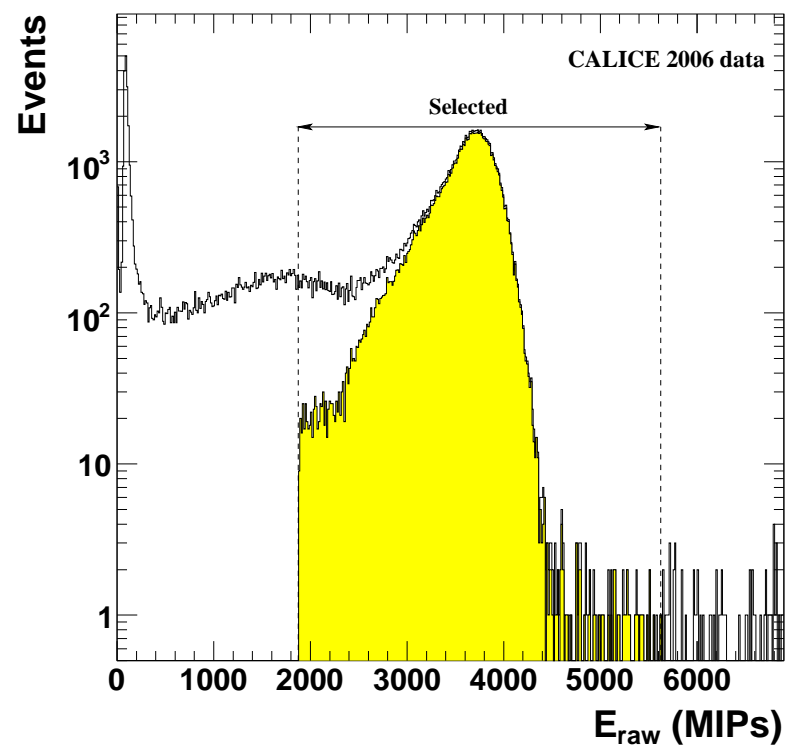

Figure 3: Distribution of total ECAL hit energies for a $15 \mathrm{GeV}$ electron run with a significant pion content. The $E_{\text {raw }}$ selection window and the shaded area obtained by demanding a signal from the Čerenkov counter are shown.

impinge in the centre of the $x$ gaps and about $20 \%$ in the case of the $y$ gap. In order to recover this loss and to have a more uniform calorimeter response, a simple method was investigated. The ECAL energy response, $f(\bar{x}, \bar{y})=E_{\text {raw }} / E_{\text {beam }}$, is measured using a combined sample of 10,15 and $20 \mathrm{GeV}$ electrons, equally populated.

The response function $f$, normalised such as to have a unit response in the middle of the wafers, is displayed on Figure 5. To characterise the $x(y)$ response, the events were required to be outside the inter-wafer gap in $y(x)$, leading to an important difference in the number of events for the two distributions, since the beam is centred on the $y$ gap. It can be parametrised with Gaussian functions, independently in $\bar{x}$ and $\bar{y}$ :

$$
f(\bar{x}, \bar{y})=\left(1-a_{x} \exp \left(-\frac{\left(\bar{x}-x_{\text {gap }}\right)^{2}}{2 \sigma_{x}^{2}}\right)\right)\left(1-a_{y} \exp \left(-\frac{\left(\bar{y}-y_{\text {gap }}\right)^{2}}{2 \sigma_{y}^{2}}\right)\right)
$$

Here, $x_{\text {gap }}$ and $y_{\text {gap }}$ are the positions at the centres of the gaps in $x$ and $y$, respectively, $a_{x}\left(a_{y}\right)$ and $\sigma_{x}\left(\sigma_{y}\right)$ their respective depths and widths in the two directions. The results of the Gaussian parametrisations are given in Table 1 The gap in $x$ is shallower and wider than that in $y$, due to the staggering of the gaps in $x[\underline{3}]$.

\begin{tabular}{|c|c|c|c|}
\hline & position $(\mathrm{mm})$ & $\sigma(\mathrm{mm})$ & $a$ \\
\hline$x$ direction & -30.0 & 4.3 & 0.143 \\
\hline$y$ direction & -8.4 & 3.2 & 0.198 \\
\hline
\end{tabular}

Table 1: Gaussian parametrisation of the inter-wafer gaps.

As illustrated in Figure 6, when the energy of each shower is corrected by $1 / f$, the average energy loss in the gaps is reduced to a few percent level. The low energy tail in 


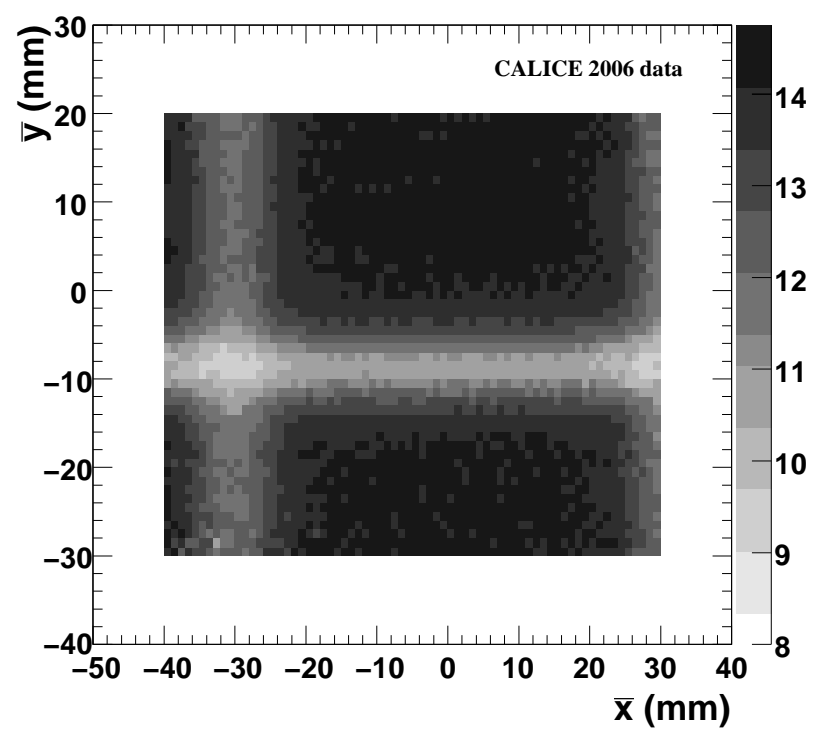

Figure 4: Mean values of $E_{\text {raw }}$ for $15 \mathrm{GeV}$ electrons as a function shower barycentre, transverse to the beam direction. The energies have been scaled down by a factor 266 to provide approximate conversion to $\mathrm{GeV}$.
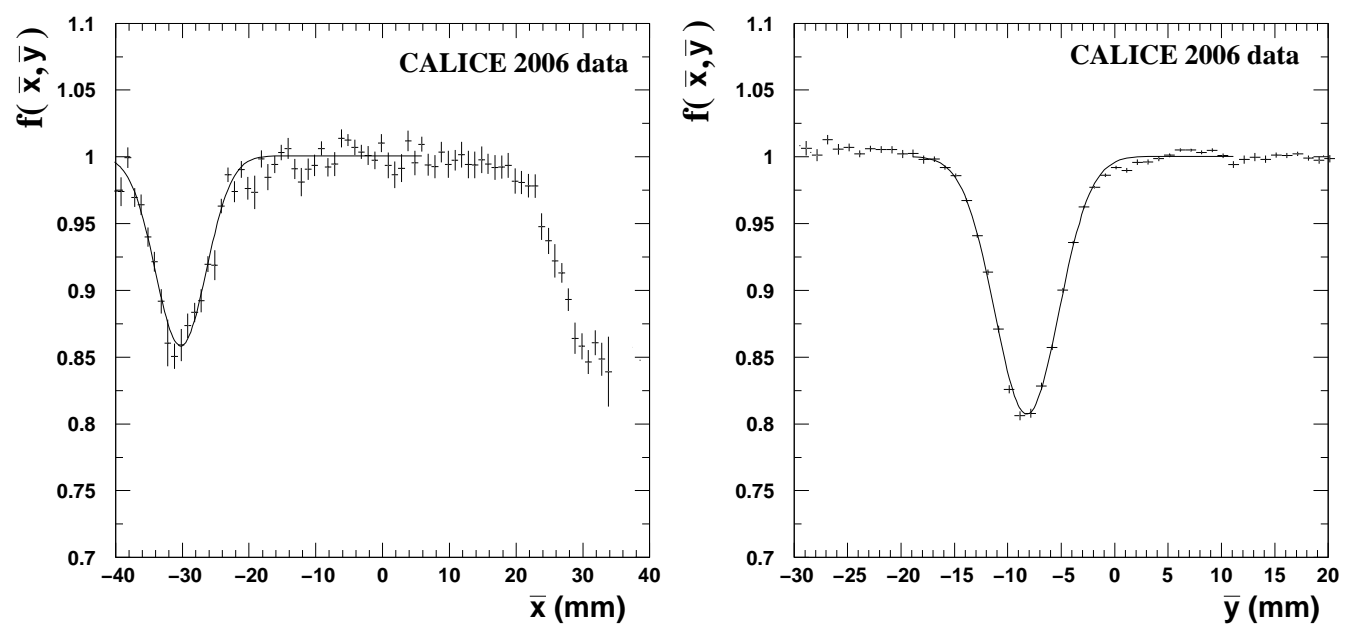

Figure 5: Normalised $f(\bar{x}, \bar{y})$ as a function of the shower barycentre coordinates, for a combined sample of 10,15 and $20 \mathrm{GeV}$ electrons. 
the energy distribution is also much reduced (Figure 17). The correction method relies only on calorimetric information and can be applied both for photons and electrons.
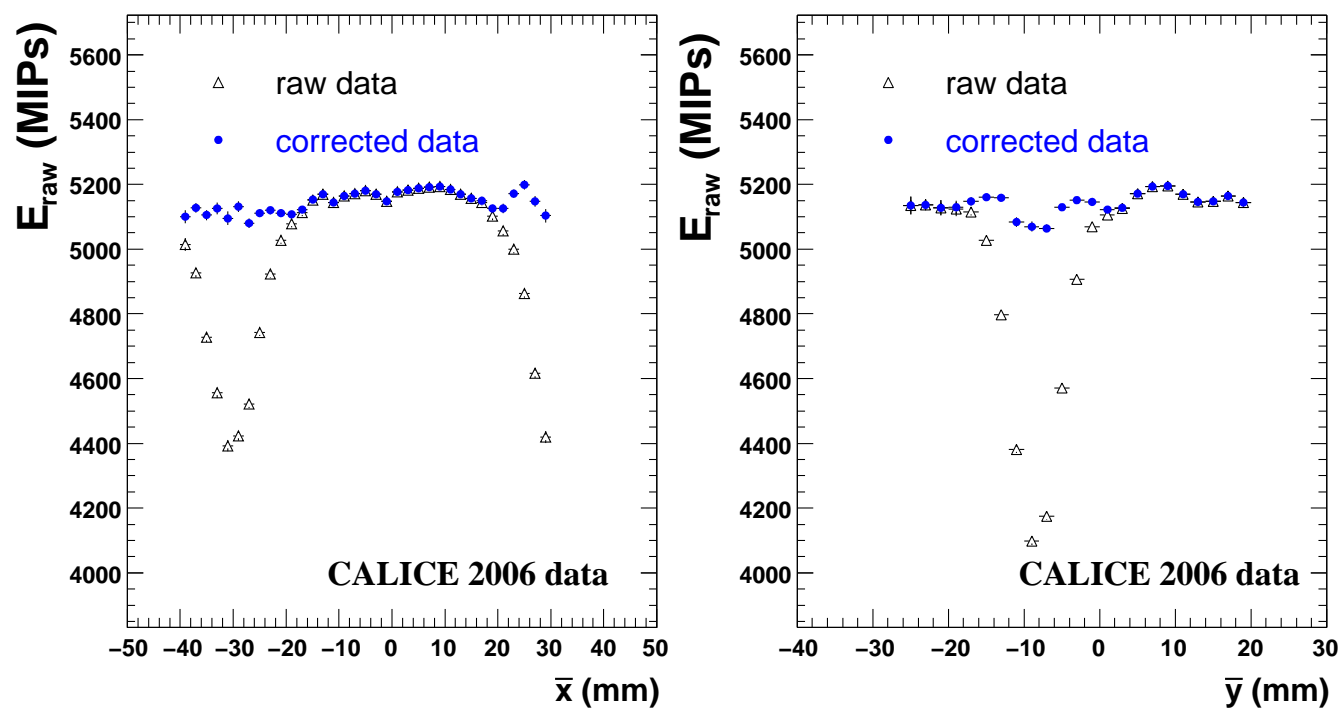

Figure 6: Mean $E_{\text {raw }}$ as $\mathrm{s}$ function of the shower barycentre coordinates for $20 \mathrm{GeV}$ electrons, before (open triangles) and after the inter-wafer gap corrections (solid circles) were applied on $E_{\text {raw }}$.

Even though it is possible to correct for the inter-wafer gaps on average, for individual events their presence will induce fluctuations in the energy response and degrade the ECAL resolution compared to a continuous calorimeter. In the data described here, the beam centre was close to the inter-wafer gap in $y$, artificially increasing the impact of the inter-wafer gaps compared to an experiment with a beam uniformely spread over the ECAL front face. Moreover, since the beam width varies strongly with the beam energy, the impact of the energy lost in the gaps is different at each energy. Therefore, in order to assess the energy response and resolution of the prototype as a function of energy in a unbiased way, only particles impinging in the middle of the wafers are selected. Since the gap effect is negligible more than 4 standard deviations away from the gap centre, the shower barycentre for selected events is required to be at a distance larger than $17.2 \mathrm{~mm}$ from the centre of the inter-wafer gap along $x$ and $12.76 \mathrm{~mm}$ away from the centre of the $y$ gap.

A sustained R\&D effort is being made to reduce the non-active areas, both by reducing the size of the inter-wafer gaps and by increasing the size of the wafers. The next SiW ECAL prototype will have $9 \times 9$ Si pads in a wafer which leads to a significant decrease of the non-active areas.

\subsection{Selection of showers well contained in the ECAL}

The fiducial volume in which the showers are fully contained in the ECAL was estimated using electrons away from the inter-wafer gaps and pointing at the centre of the ECAL. The radial shape of an average $45 \mathrm{GeV}$ electron shower is shown in Figure 8 , both for data and simulation. The simulation reproduces the shower width to better 


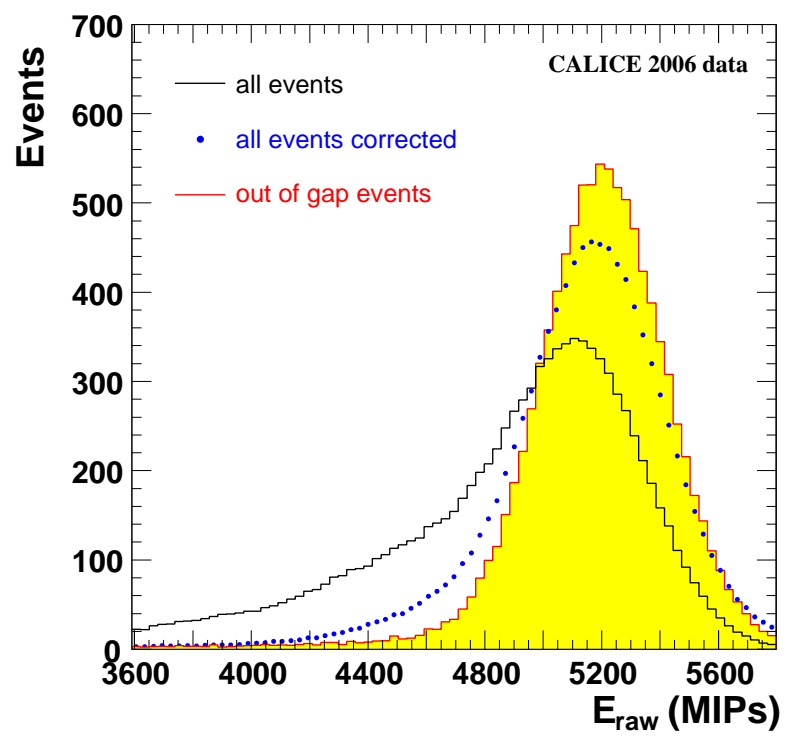

Figure 7: Energy distribution for $20 \mathrm{GeV}$ electrons in the cases of: events outside the inter-wafer gaps (solid histogram), all events without inter-wafer gap corrections (open histogram) and all events with inter-wafer gap corrections (solid circles). The histograms are normalised to the same number of entries.

than 2\%: $95 \%$ of the shower energy is contained within $30.5 \mathrm{~mm}$ (i.e. less than four pads), to be compared with $29.9 \mathrm{~mm}$ in the case of the simulated showers. To ensure radial containment, all electrons impinging on the ECAL front face less than $32 \mathrm{~mm}$ from one of the ECAL borders are therefore excluded from the selected sample.

The longitudinal containment of the showers is ensured by rejecting events which have the maximum of the energy deposited along the $z$ direction in the first five layers or the last five layers of the prototype. Only $0.21 \%$ of the simulated $6 \mathrm{GeV}$ electrons fail these containment criteria and $0.02 \%$ of the $45 \mathrm{GeV}$ electrons.

\subsection{Rejection of electrons showering in front of ECAL}

The data recorded at CERN contain a significant number of events which have approximately the expected energy for a single electron, but whose spatial structure clearly exhibits double showers. A likely explanation is bremsstrahlung far upstream in the beamline. In the Monte Carlo simulation, the known material between the Cerenkov counter and the calorimeter is simulated, and yet the agreement between the rate of double shower events is poor between simulations and data. Before comparing data and Monte Carlo, it is therefore necessary to select a sample of single electron showers. To this end, the energy deposits in the shower are projected in a two-dimensional histogram, on the transverse, $x-y$, plane. The binning of the histogram is the same in $x$ and $y$ and corresponds to the cell size $(1 \mathrm{~cm})$. A simple nearest-neighbour clustering algorithm (including diagonal neighbours) is applied on the bins with energies above a given threshold $T$, in order to select events with more than one local maximum for the energy deposit. For each event we determine the maximum value of the threshold, $T_{\max }$, above which the event would be reconstructed as a single cluster. In Figure 9 we compare the distribu- 


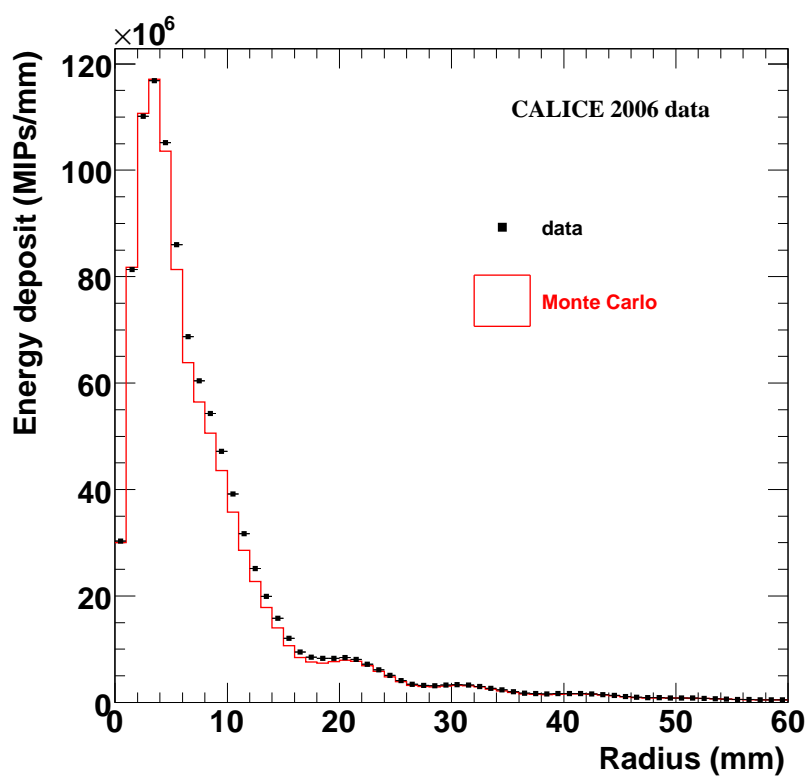

Figure 8: Energy deposited in ECAL as a function of the radial distance to the longitudinal shower axis, integrated over 171671 showers of $45 \mathrm{GeV}$.

tions of $T_{\max }$ between data and simulation for a $30 \mathrm{GeV}$ beam. A sizeable discrepancy is seen for larger values of $T_{\max }$ and therefore a cut is applied on $T_{\max }$. The cut is energy dependent, varying from $50 \mathrm{MIPs}$ at $10 \mathrm{GeV}$ to $120 \mathrm{MIPs}$ at $45 \mathrm{GeV}$. This cut typically rejects $\sim 20 \%$ of data and $\sim 2-3 \%$ of simulated events at the higher energies.

A summary of the selected electron and positron data is shown in Table 2. The number of simulated events available for each energy is also indicated.

\begin{tabular}{|c|c|c|c|}
\hline Energy $(\mathrm{GeV})$ & date & data statistics (kevts) & MC statistics (kevts) \\
\hline 6 & Oct & 6.6 & 83.2 \\
\hline 10 & Aug, Oct & 43.1 & 80.3 \\
\hline 12 & Oct & 27.2 & 72.8 \\
\hline 15 & Aug, Oct & 51.4 & 70.3 \\
\hline 20 & Aug & 62.9 & 56.2 \\
\hline 30 & Aug & 42.3 & 55.2 \\
\hline 40 & Aug & 22.9 & 67.8 \\
\hline 45 & Aug & 108.6 & 108.8 \\
\hline
\end{tabular}

Table 2: Summary of the electron events selected for this analysis. 


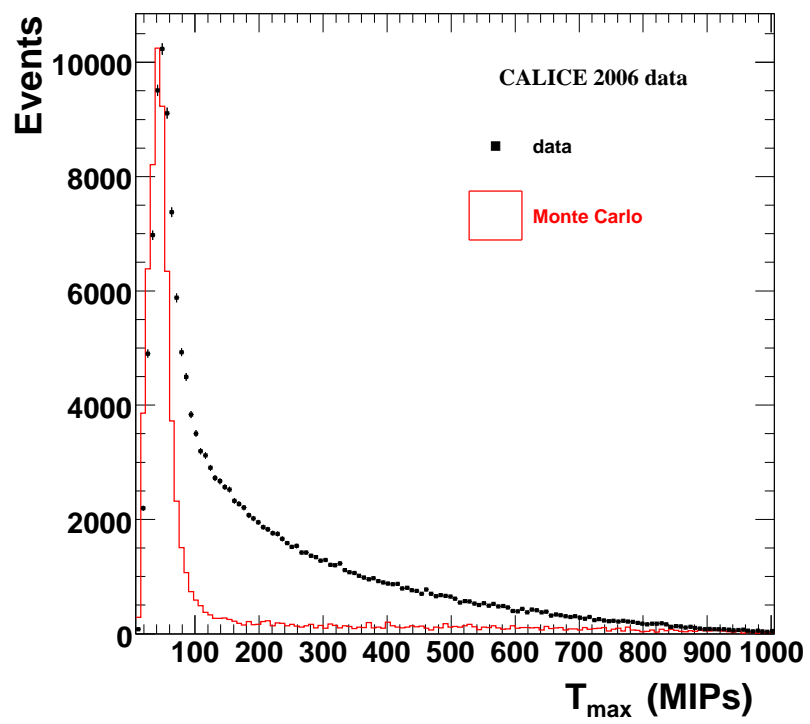

Figure 9: Distribution of the variable $T_{\max }$ (described in the text), which is used to reduce the contribution of double showers. Data and simulation are compared at $30 \mathrm{GeV}$.

\section{Performance Studies}

\subsection{ECAL Sampling Fraction Scheme}

The ECAL is made of 30 layers grouped in three modules of 10 layers each [3]. Each tungsten sheet has the same thickness in a given module. However, as can be derived from Figure 10, where one passive tungsten layer sandwiched between two active silicon layers is shown, two successive silicon layers are either separated by one thickness of tungsten or by the same thickness of tungsten plus two thicknesses of PCB, aluminium and carbon-fibre-epoxy composite. A different sampling fraction, defined as the ratio of the energy deposited in the active medium to the total energy deposit (sum of the energy deposits in the active and passive medium), is therefore expected for the even and the odd layers of the same calorimeter module.

The easiest method to investigate this difference is to compare in each module the mean energy deposits in odd and even layers. For the first module, if we neglect the shower profile, the ratio of the two is

$$
R=\frac{E^{\text {odd }}}{E^{\text {even }}}=1+\eta
$$

with $\eta$ being, approximately, the ratio of the non-tungsten radiation length to the tungsten radiation length.

When counting the layers starting from zero, the odd layers are systematically shifted compared to the even layers towards the shower maximum and the measurement of $R$ is biased by the shower development. To overcome this bias, $R$ is measured twice, either comparing the odd layers with the average of the surrounding even layers, or comparing 


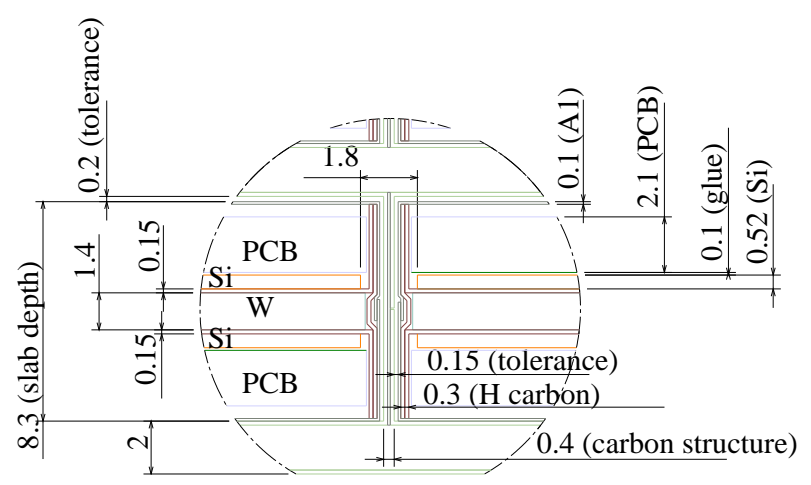

Figure 10: Details of one ECAL slab, showing one passive tungsten layer sandwiched between two active silicon layers. The dimensions are in $\mathrm{mm}$. In contrast to the upper silicon layer which is preceded by a layer of tungsten only, the lower silicon layer is preceded by a larger passive layer: the PCB, aluminium, glue and carbon structure as well as the tungsten.

the even layers with the average of the neighbouring odd layers:

$$
\begin{aligned}
R^{\prime} & =\frac{\left\langle\mathrm{E}_{1}+\mathrm{E}_{3}+\mathrm{E}_{5}+\mathrm{E}_{7}\right\rangle}{\left\langle\frac{\mathrm{E}_{0}+\mathrm{E}_{2}}{2}+\frac{\mathrm{E}_{2}+\mathrm{E}_{4}}{2}+\frac{\mathrm{E}_{4}+\mathrm{E}_{6}}{2}+\frac{\mathrm{E}_{6}+\mathrm{E}_{8}}{2}\right\rangle} \\
R^{\prime \prime} & =\frac{\left\langle\frac{\mathrm{E}_{1}+\mathrm{E}_{3}}{2}+\frac{\mathrm{E}_{3}+\mathrm{E}_{5}}{2}+\frac{\mathrm{E}_{5}+\mathrm{E}_{7}}{2}+\frac{\mathrm{E}_{7}+\mathrm{E}_{9}}{2}\right\rangle}{\left\langle\mathrm{E}_{2}+\mathrm{E}_{4}+\mathrm{E}_{6}+\mathrm{E}_{8}\right\rangle}
\end{aligned}
$$

where $\mathrm{E}_{n}$ is the energy deposit in the layer number $n$ and the brackets indicate that mean values are used. The value of $\eta$ is taken as the average of $R^{\prime}-1$ and $R^{\prime \prime}-1$, while the difference between them gives a conservative estimate of the systematic uncertainty due to the shower shape. As an example, the distributions of the energy deposits in the odd and even layers are shown in Figure 11 for $20 \mathrm{GeV}$ electrons.

The values of $\eta$, obtained using the first module and for different beam energies, are displayed in Figure 12. The overall value is $(7.2 \pm 0.2 \pm 1.7) \%$. The measurement of $\eta$ using the second and third module gives compatible results and the corresponding value obtained from simulation is $(4.7 \pm 0.2 \pm 2.0) \%$.

In computing the total response of the calorimeter, the sampling fraction for layer $i$ is given by $w_{i}=K$ for even layers and $w_{i}=K+\eta$ for the odd layers, with $K=1,2,3$ in modules $1,2,3$, respectively.

\subsection{Linearity and energy resolution}

The total response of the calorimeter is calculated as

$$
E_{\mathrm{rec}}(\mathrm{MIPs})=\sum_{i} w_{i} E_{i}
$$

with $w_{i}$ the sampling fraction for the layer $i$. Its distribution for electrons at $30 \mathrm{GeV}$ is shown in Figure 13, together with a fit using a Gaussian function in the range $[-\sigma,+2 \sigma]$. There is reasonably good agreement between data and simulation. An asymmetric range is chosen for the fit in order to reduce sensitivity to pion background, to radiative effects 


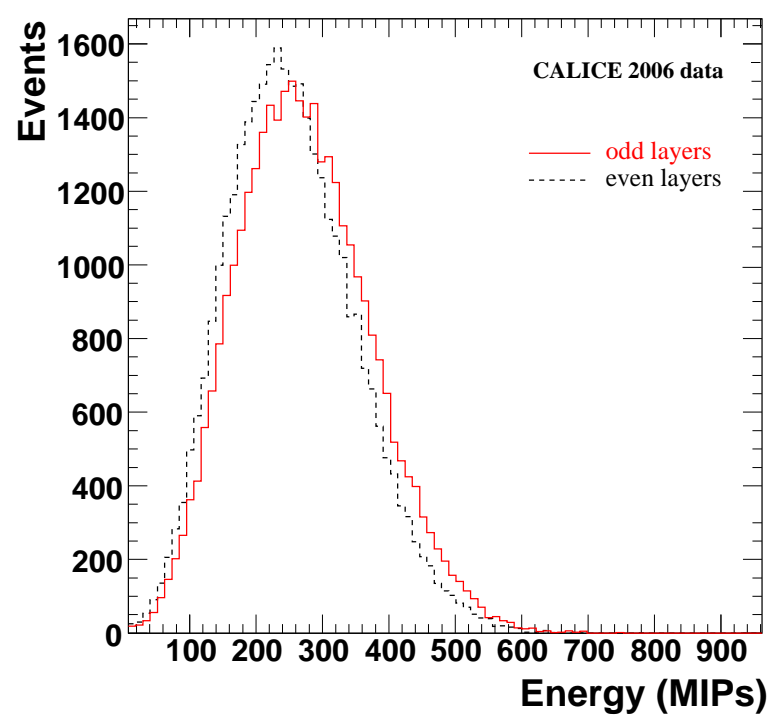

Figure 11: Energy deposits in odd layers (continuous histogram) and average energy deposits in their surrounding even layers (dashes) by $20 \mathrm{GeV}$ electrons, in the first ECAL module.

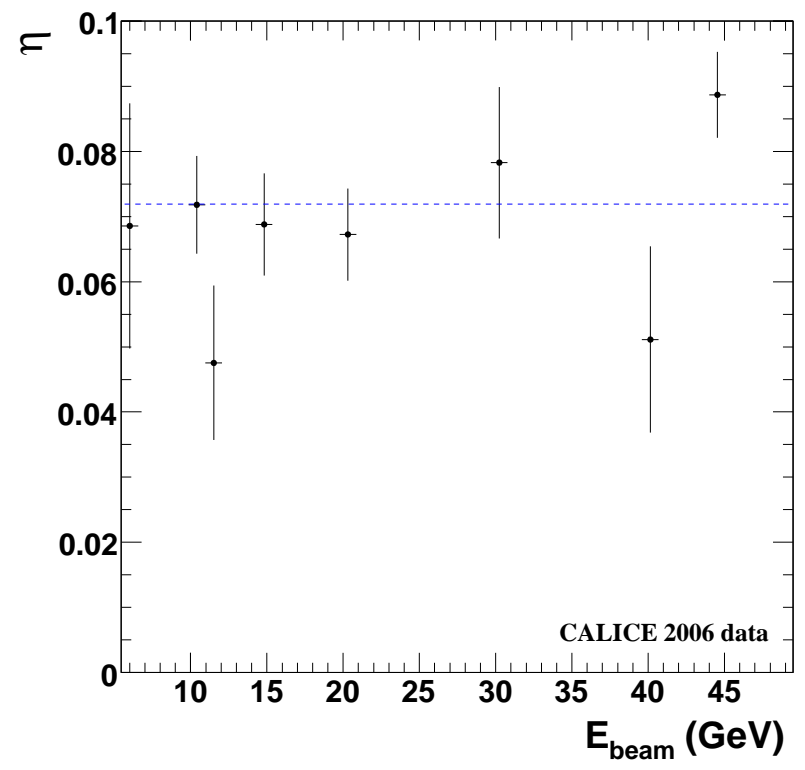

Figure 12: Values of $\eta$ as a function of the beam energy. The uncertainties are statistical and the dashed line gives the average value of $\eta$. 
upstream of the calorimeter, and to any residual influence of the inter-wafer gaps. The position of the peak is the mean energy response (called in the following $E_{\text {mean }}$ ) and its distribution is shown in Figure 14 as a function of the beam energy. The uncertainties on $E_{\text {mean }}$ are those estimated from the fit.

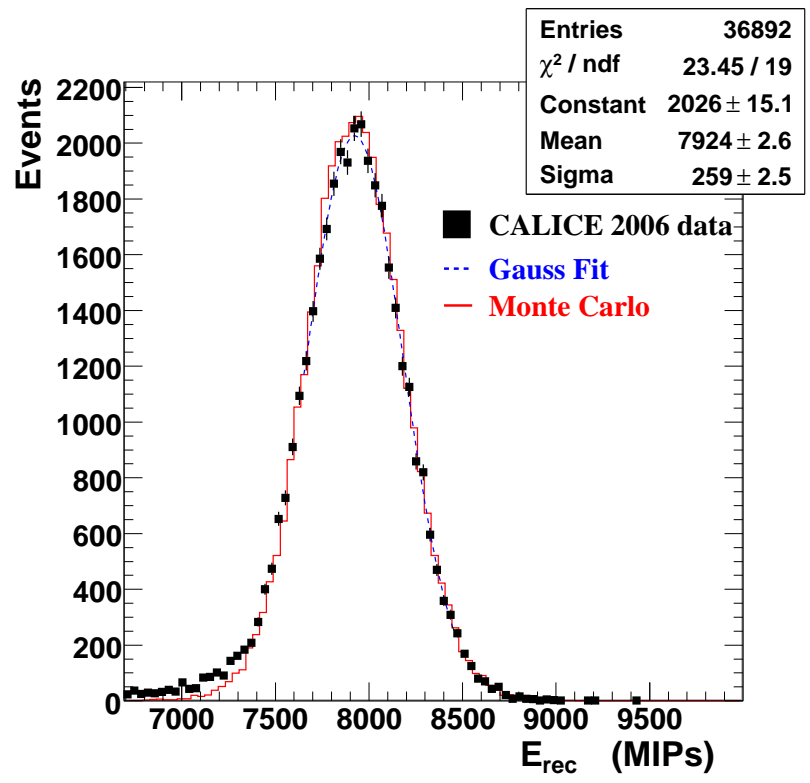

Figure 13: Gaussian parametrisation of $E_{\mathrm{rec}}$ for $30 \mathrm{GeV}$ electron data (dashes). The range of the fit is $[-\sigma,+2 \sigma]$. The data (solid squares) and simulation (continuous line) are superposed.

From the dispersion of $E_{\text {mean }}$ in the different runs at the same nominal beam energy, the uncertainty of the beam mean energy, $E_{\text {beam, }}$ was estimated to be

$$
\frac{\Delta E_{\text {beam }}}{E_{\text {beam }}}=\frac{0.12}{E_{\text {beam }}(\mathrm{GeV})} \oplus 0.1 \%,
$$

The first term is related to hysteresis in the bending magnets, while the calibration and the uncertainties on the collimator geometry give the constant term. For comparison, in [9], the uncertainty on the beam mean energy was quoted as

$$
\frac{\Delta E_{\text {beam }}}{E_{\text {beam }}}=\frac{0.25}{E_{\text {beam }}(\mathrm{GeV})} \oplus 0.5 \%,
$$

The first of these parametrisations of the uncertainty (Equation 9) is used in the following, except for checks of systematic uncertainties.

The mean energy response can be parametrised as $E_{\text {mean }}=\beta \cdot E_{\text {beam }}-\alpha$, while the measured energy $E_{\text {meas }}$ is given by $E_{\text {meas }}=E_{\text {mean }}+\alpha$. The parameter $\beta$ is a global MIP to $\mathrm{GeV}$ calibration factor. The offset $\alpha$ is partly due to the rejection of the low energy hits and it increases steadily with the hit energy threshold, as displayed on Figure 15. On the same figure are also shown the values of the offset, as expected from simulation. The bias introduced by the fact that the uncertainty on the beam mean energy decreases 


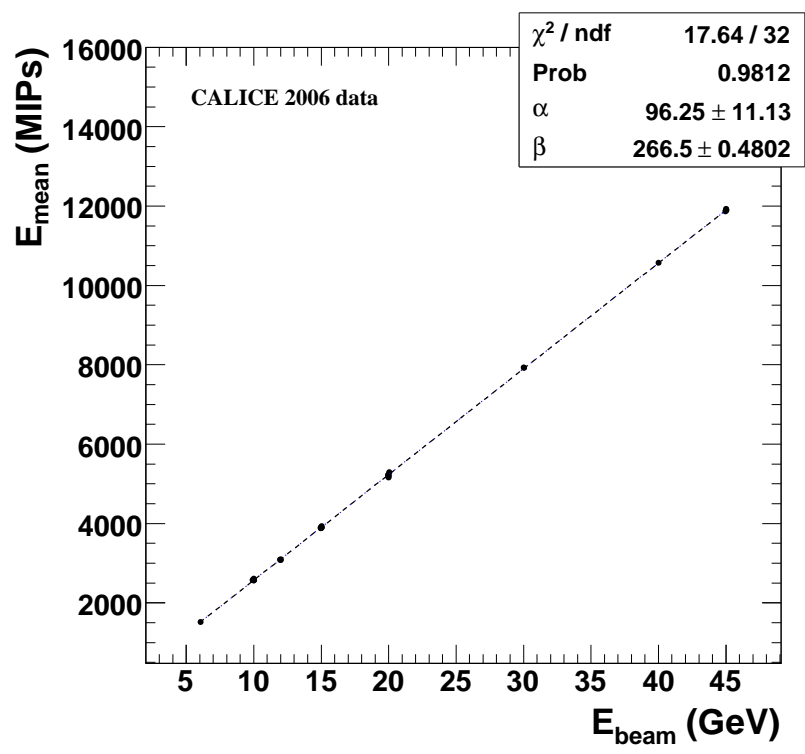

Figure 14: Energy response of the ECAL as a function of the beam energy. For clarity, all the runs around the same nominal energy of the beam were combined in one entry for the plot, for which entry the uncertainty was estimated assuming that the uncertainties on the individual runs were uncorrelated.

with increasing beam energy and enhances therefore the weight of the high energy runs was estimated by artificially assigning to the simulated data uncertainties on $E_{\text {beam }}$ according to Equation 9. By taking into account this bias, the disagreement between data and simulation is somewhat reduced (Figure 15).

The residuals to linearity of the measured energy, converted from MIPs to GeV using a constant $266 \mathrm{MIPs} / \mathrm{GeV}$ conversion factor (obtained from Figure 14), are shown in Figure [16 as a function of the beam energy. The residuals are within approximately the $1 \%$ level and are consistent with zero non-linearity. Data and simulation agree within one standard deviation.

The relative energy resolution, $\Delta E_{\text {meas }} / E_{\text {meas }}$, as shown in Figure 17, can be parametrised by a quadrature sum of stochastic and constant terms

$$
\frac{\Delta E_{\text {meas }}}{E_{\text {meas }}}=\left(\frac{16.6 \pm 0.1}{\sqrt{E(\mathrm{GeV})}} \oplus(1.1 \pm 0.1)\right) \%,
$$

where the intrinsic momentum spread of the beam was subtracted from the ECAL data [6]. By varying the range for fitting $E_{\text {rec }}$, the variation of the stochastic term has the same order of magnitude as the statistical error, whereas the constant term remains stable: a fitting interval reduced to $[-0.75 \sigma,+1.75 \sigma]$ improves the stochastic term to $16.5 \pm 0.2$, whereas an enlargement to $[-2 \sigma,+3 \sigma]$ degrades it to $16.7 \pm 0.1$. The expected resolution from simulation is

$$
\left[\frac{\Delta E_{\text {meas }}}{E_{\text {meas }}}\right]^{\mathrm{MC}}=\left(\frac{17.3 \pm 0.1}{\sqrt{E(\mathrm{GeV})}} \oplus(0.5 \pm 0.1)\right) \%
$$




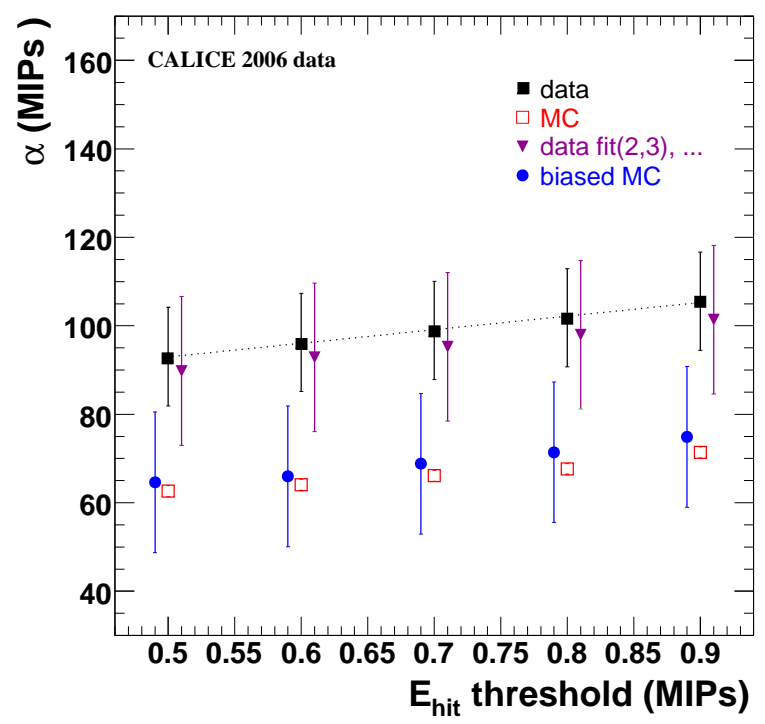

Figure 15: Variation of the linearity offset with the hit energy threshold: data (solid squares), data with the fitting range of $E_{\mathrm{rec}}$ enlarged to $[-2 \sigma,+3 \sigma]$ and considering the parametrisation in Equation 10 for the uncertainty on the beam mean energy (solid triangles), Monte Carlo (open squares), Monte Carlo with an artificial uncertainty on the beam mean energy as given by Equation 10 (solid circles). For clarity, the points were artificially shifted along the $x$ axis around the nominal $E_{\text {hit }}$ threshold.

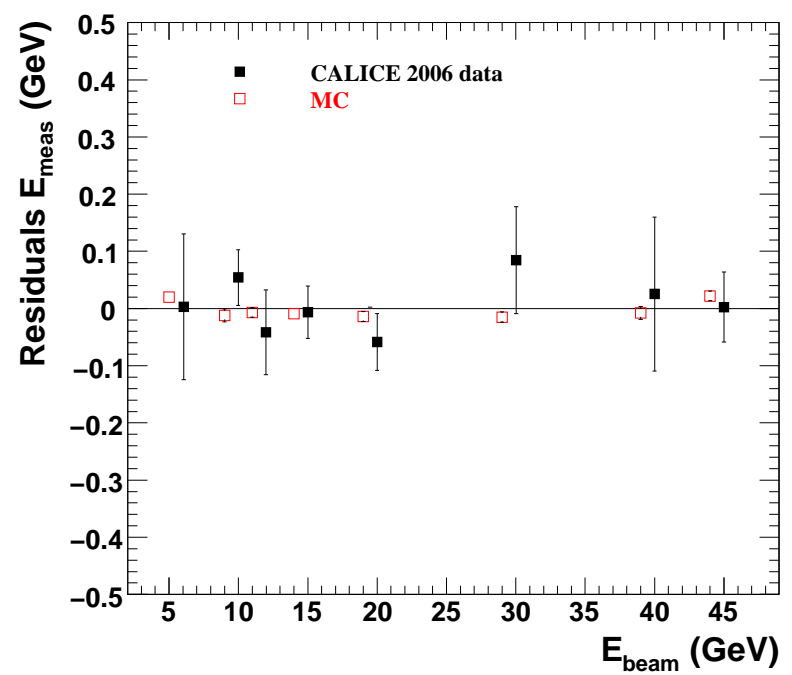

Figure 16: Residuals to linearity of $E_{\text {meas }}$ as a function of the beam energy, for data (solid squares) and simulation (open squares). All the runs around the same nominal energy of the beam were combined in one entry, for which the uncertainty was estimated assuming that the uncertainties on the individual runs were uncorrelated. For clarity, the Monte Carlo points were artificially shifted along the $x$ axis around the nominal $E_{\text {beam }}$. 
which agrees within $5 \%$ with the measured resolution of the prototype.

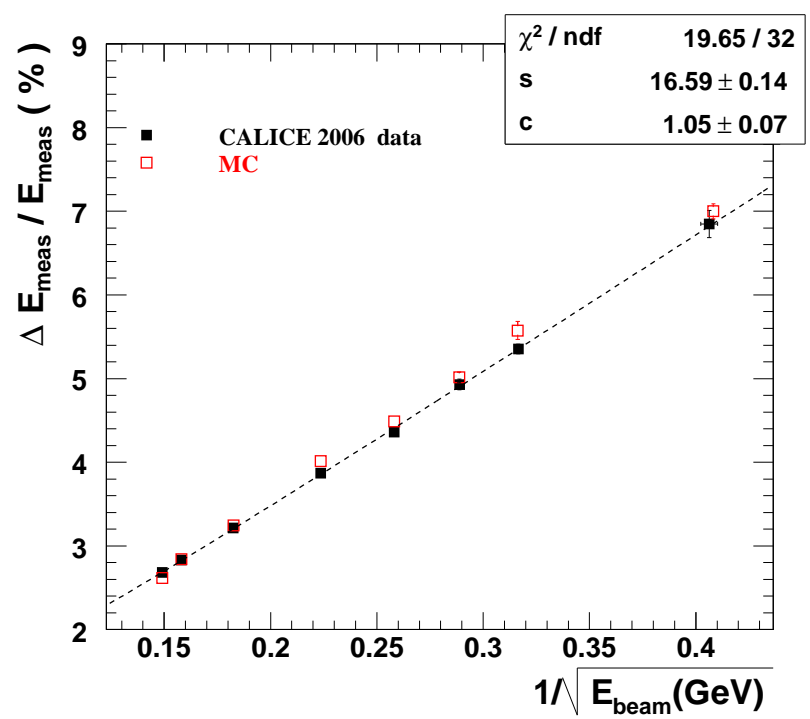

Figure 17: Relative energy resolution $\left(\Delta E_{\text {meas }} / E_{\text {meas }}\right)$ as a function of the beam energy (solid squares), and its usual parametrisation as $s / \sqrt{E} \oplus c$. For clarity, the 35 runs available were combined into 8 different beam energy points for the plot. For the parametrisation of the energy resolution each run was however treated individually. The values expected from simulation are superposed (open squares).

Different systematic checks have been performed on the data. Variations of the linearity and resolution against the minimal accepted distance between the shower barycentre and the nearest inter-wafer gap, when the energy threshold for considering the hits is 0.6 MIPs are shown in Table 3. In addition, this hit energy threshold has itself been varied (Table 4). In order to investigate the potential effects linked to the beam position, the energy response is also compared for showers with barycentres located in the right hand side (negative $x$ coordinates) and in the upper half of the detector (upper row of wafers) as summarised in Table [5. The results of all checks are consistent. Since data were taken in both August and October 2006, it was also possible to check the response stability in time and no significant differences between the two data samples are observed.

\section{Conclusion}

The response to normally incident electrons of the CALICE Si-W electromagnetic calorimeter was measured for energies between 6 and $45 \mathrm{GeV}$, using the data recorded in 2006 at CERN.

The calorimeter response is linear to within approximately $1 \%$. The energy resolution has a stochastic term of $(16.6 \pm 0.1) \% / \sqrt{E(\mathrm{GeV})}$, whereas the constant term is $1.1 \pm$ $0.1 \%$. Several sources of systematic uncertainties have been investigated and their effect is within the statistical uncertainties. The agreement between data and Monte Carlo simulation is within $5 \%$. 


\begin{tabular}{|c|c|c|c|c|}
\hline \multirow{2}{*}{} & \multicolumn{4}{|c|}{ shower distance to the gaps (in standard deviations) } \\
\cline { 2 - 5 } & 3.5 & 4 & 4.5 & 5 \\
\hline $\begin{array}{c}\chi^{2} / n d f \\
\text { (linearity) }\end{array}$ & $16.8 / 32$ & $17.6 / 32$ & $18.9 / 32$ & $24.2 / 32$ \\
\hline $\begin{array}{c}\alpha \\
(\text { MIPs })\end{array}$ & $93.9 \pm 11.1$ & $96.3 \pm 11.2$ & $97.8 \pm 11.5$ & $99.1 \pm 11.6$ \\
\hline $\begin{array}{c}\beta \\
(\text { MIPs/GeV) }\end{array}$ & $266.3 \pm 0.5$ & $266.6 \pm 0.5$ & $266.8 \pm 0.5$ & $266.8 \pm 0.5$ \\
\hline $\begin{array}{c}\text { resolution (\%) } \\
(\text { stochastic term) }\end{array}$ & $16.7 \pm 0.1$ & $16.6 \pm 0.1$ & $16.4 \pm 0.2$ & $16.3 \pm 0.2$ \\
\hline $\begin{array}{c}\text { resolution (\%) } \\
\text { constant term) }\end{array}$ & $1.0 \pm 0.1$ & $1.0 \pm 0.1$ & $1.1 \pm 0.1$ & $1.2 \pm 0.1$ \\
\hline
\end{tabular}

Table 3: Impact of the distance of shower to the inter-wafer gaps on the ECAL linearity and resolution. The distance is given in terms of standard deviations to the gap centre, with the standard deviation defined by the Gaussian parametrisation of the gaps.

\begin{tabular}{|c|c|c|c|}
\hline & \multicolumn{3}{|c|}{$E_{\text {hit }}$ cutoff (MIPs) } \\
\cline { 2 - 4 } & 0.5 & 0.7 & 0.9 \\
\hline $\begin{array}{c}\chi^{2} / n d f \\
\text { (linearity) }\end{array}$ & $18.0 / 32$ & $17.8 / 32$ & $18.0 / 32$ \\
\hline $\begin{array}{c}\alpha \\
(\text { MIPs) }\end{array}$ & $93.0 \pm 11.2$ & $98.9 \pm 11.1$ & $105.6 \pm 11.1$ \\
\hline $\begin{array}{c}\beta \\
(\text { MIPs/GeV) }\end{array}$ & $266.8 \pm 0.5$ & $266.3 \pm 0.5$ & $265.8 \pm 0.5$ \\
\hline $\begin{array}{c}\text { resolution (\%) } \\
\text { (stochastic term) }\end{array}$ & $16.6 \pm 0.1$ & $16.5 \pm 0.1$ & $16.6 \pm 0.1$ \\
\hline $\begin{array}{c}\text { resolution (\%) } \\
\text { constant term) }\end{array}$ & $1.0 \pm 0.1$ & $1.1 \pm 0.1$ & $1.1 \pm 0.1$ \\
\hline
\end{tabular}

Table 4: Impact of the hit energy cutoff on the ECAL linearity and resolution.

\section{Acknowledgements}

We would like to thank the technicians and the engineers who contributed to the design and construction of the prototypes, including U. Cornett, G. Falley, K Gadow, P. Göttlicher, S. Karstensen and P. Smirnov. We also gratefully acknowledge the DESY and CERN managements for their support and hospitality, and their accelerator staff for the reliable and efficient beam operation. We would like to thank the HEP group of the University of Tsukuba for the loan of drift chambers for the DESY test beam. The authors would like to thank the RIMST (Zelenograd) group for their help and sensors manufacturing. This work was supported by the Bundesministerium für Bildung und Forschung, Germany; by the DFG cluster of excellence "Origin and Structure of the Universe"; by the Helmholtz-Nachwuchsgruppen grant VH-NG-206; by the BMBF, grant numbers 05HS6VH1 and 05HS6GU1; by the Alexander von Humboldt Foundation (Research Award IV, RUS1066839 GSA); by joint Helmholtz Foundation and RFBR 


\begin{tabular}{|c|c|c|}
\hline & right side & upper part \\
\hline$\alpha(\mathrm{MIPs})$ & $96.1 \pm 10.9$ & $97.7 \pm 11$ \\
\hline$\beta(\mathrm{MIPs} / \mathrm{GeV})$ & $266.6 \pm 0.5$ & $266.8 \pm 0.5$ \\
\hline resolution (stochastic term) $(\%)$ & $16.8 \pm 0.1$ & $16.8 \pm 0.2$ \\
\hline resolution (constant term) $(\%)$ & $1.1 \pm 0.1$ & $1.1 \pm 0.1$ \\
\hline
\end{tabular}

Table 5: Response to electrons crossing the right hand side and the upper part of the ECAL.

grant HRJRG-002, Russian Agency for Atomic Energy, ISTC grant 3090; by Russian Grants SS-1329.2008.2 and RFBR0402/17307a and by the Russian Ministry of Education and Science; by CRI(MST) of MOST/KOSEF in Korea; by the US Department of Energy and the US National Science Foundation; by the Ministry of Education, Youth and Sports of the Czech Republic under the projects AV0 Z3407391, AV0 Z10100502, LC527 and by the Grant Agency of the Czech Republic under the project 202/05/0653; and by the Science and Technology Facilities Council, UK.

\section{References}

[1] T. Behnke et al (ed.), Reference Design Report "Volume 4: Detectors", available at \protect\vrulewidth0pthttp://lcdev.kek.jp/RDR

[2] J.-C. Brient, "Improving the jet reconstruction with the particle flow method: An introduction", in the Proceedings of 11th International Conference on Calorimetry in High-Energy Physics (Calor 2004), Perugia, Italy, March 2004.

[3] M. Anduze et al, "Design and Commissioning of the Physics Prototype of a Si-W Electromagnetic Calorimeter for the International Linear Collider", JINST 3 (2008) P08001, 33p.

[4] F. Sefkow, "The scintillator HCAL testbeam prototype", in the Proceedings of 2005 International Linear Collider Workshop (LCWS 2005), Stanford, California, March 2005.

[5] D. Chakraborty, "The Tail-Catcher/Muon Tracker for the CALICE Test Beam"', in the Proceedings of 2005 International Linear Collider Workshop (LCWS 2005), Standford, California, March 2005.

[6] Description of the CERN H6 testbeam area available at \protect \vrulewidth0pthttp://ab-div-atb-ea.web.cern.ch/ab-div-atb-ea/BeamsAndAreas/

[7] "Mokka Geant4 Application for Linear Collider Detectors", see \protect \vrulewidth0pthttp://polzope.in2p3.fr:8081/MOKKA

[8] S. Agostinelli et al, "Geant4 - A Simulation Toolkit", NIM A 506 (2003) 250-303.

[9] S. Akhmadaliev et al, "Results from a new combined test of an electromagnetic liquid argon calorimeter with a hadronic scintillating-tile calorimeter", NIM A 449 (2000) 461-477. 